\title{
Radiosensitizing effect of 5-aminolevulinic acid-induced protoporphyrin IX in glioma cells in vitro
}

\author{
JUNKOH YAMAMOTO ${ }^{1}$, SHUN-ICHIRO OGURA ${ }^{2}$, TOHRU TANAKA ${ }^{3}$, TAKEHIRO KITAGAWA ${ }^{1}$, \\ YOSHITERU NAKANO ${ }^{1}$, TAKESHI SAITO ${ }^{1}$, MAYU TAKAHASHI $^{1}$, \\ DAISUKE AKIBA $^{1}$ and SHIGERU NISHIZAWA ${ }^{1}$ \\ ${ }^{1}$ Department of Neurosurgery, University of Occupational and Environmental Health, Fukuoka; \\ ${ }^{2}$ Endowed Research Section (ALA), Frontier Research Center, Tokyo Institute of \\ Technology, Yokohama; ${ }^{3}$ SBI ALApromo Co., Ltd., Tokyo, Japan
}

Received December 5, 2011; Accepted January 18, 2012

DOI: 10.3892/or.2012.1699

\begin{abstract}
Aminolevulinic acid (ALA) is a prodrug used in photodynamic therapy and fluorescence-guided resection of malignant gliomas due to its high cellular uptake in tumours. Porphyrin compounds act not only as photosensitizers but also as radiosensitizers. In the present study, the possible use of 5-ALA as a radiosensitizer for malignant gliomas was examined in vitro. Rat glioma cell lines (9L, C6) were pre-treated with 5-ALA and exposed to ionizing irradiation. The radiosensitizing effect of 5-ALA was evaluated by colony-forming assay. Intracellular reactive oxygen species (ROS) produced by 5-ALA and irradiation were evaluated by confocal laser scanning microscopy. Pre-treatment with 5-ALA enhanced the radiosensitivity of $9 \mathrm{~L}$ cells to single-dose ionizing irradiation compared with controls $\left(\mathrm{D}_{0}\right.$ value, $4.35 \pm 0.20$ and $4.84 \pm 0.23$ Gy, respectively, $\mathrm{P} \leq 0.05)$. Exposure to multi-dose ionizing irradiation revealed high radiosensitivity in both $9 \mathrm{~L}$ and C6 cells pre-treated with 5-ALA compared to controls. Production of intracellular ROS increased in 9L cells pretreated with 5-ALA after ionizing irradiation compared to control cells. Thus, 5-ALA functions as a specific radiosensitizer for malignant gliomas. Intracellular 5-ALA-induced PpIX plays an important role in the production of ROS and the radiosensitizing effect under ionizing irradiation conditions.
\end{abstract}

\section{Introduction}

Malignant gliomas are highly aggressive tumours characterized by a recurrence rate of virtually $100 \%$, despite several treat-

Correspondence to: Dr Junkoh Yamamoto, Department of Neurosurgery, University of Occupational and Environmental Health, Iseigaoka, Yahatanishi-ku, Kitakyushu, Fukuoka 807-8555, Japan

E-mail: yama9218@med.uoeh-u.ac.jp

Key words: glioblastoma, free radical, photosensitizer, imaging, radiation therapy, malignancy ment options, including aggressive surgical resection, radiation therapy and chemotherapy $(1,2)$. Photodynamic therapy (PDT) is an effective treatment method for several kinds of neoplasms and is based on the use of different photosensitizers (3-6). In general, the effectiveness of a photosensitizer depends on two important properties, namely the production of cytotoxic oxygen and fluorescence excited by laser irradiation, which are important for PDT and fluorescence-guided resection in neurosurgery (7-9). Recent studies revealed the radiosensitizing effects of porphyrin compounds such as Photofrin and haematoporphyrin derivatives (HpD) (10-12). However, to date, few studies have provided evidence of the effect of 5-aminolevulinic acid (ALA)-induced porphyrin IX (PpIX) as radiosensitizer. Furthermore, the radiosensitizing effect of 5-ALA-induced PpIX is controversial (13-15).

In the present study, the radiosensitizing effect of 5-ALA-induced PpIX was investigated in glioma cells in vitro. The cytotoxic effect of combined treatment with 5-ALA and ionizing irradiation was evaluated by colony-forming assays in glioma cells. The formation of reactive oxygen species (ROS) in response to this combined treatment was assessed in glioma cells using confocal laser scanning microscopy. The effectiveness of 5-ALA-induced PpIX as a radiosensitizer is also discussed.

\section{Materials and methods}

Chemicals. 5-ALA was purchased from Cosmobio K.K. (Tokyo, Japan) and dissolved in fresh culture medium at a final concentration of $1 \mathrm{mM}$ for the in vitro study. 2',7'-Dichlorofluorescein diacetate (DCFD) was purchased from Sigma-Aldrich Japan K.K. (Tokyo, Japan). DCFD was dissolved in fresh culture medium at a final concentration of $10 \mu \mathrm{M}$. Other materials were of the highest grade available.

Cell culture. Two different rat glioma cell lines (9L gliosarcoma and C6 glioma) were used in this study. 9L gliosarcoma and C6 glioma cells were cultured for several days in RPMI-1640 and Dulbecco's modified Eagle's medium (DMEM) with 10\% fetal bovine serum at $37^{\circ} \mathrm{C}$ before use, respectively. Both cell 
lines were maintained in a humidified incubator with $5 \% \mathrm{CO}_{2}$ at $37^{\circ} \mathrm{C}$. Cells were passaged in the exponential growing phase using a $0.05 \%$ trypsin solution containing $0.5 \mathrm{mM}$ EDTA.

Evaluation of the response of cells to single-dose ionizing irradiation. The 9L gliosarcoma and C6 glioma cells were seeded at a density of 100 cells ( 0 and 2 Gy), 200 cells ( 4 Gy), 400 cells (6 and $8 \mathrm{~Gy}$ ) and 600 cells (10 Gy) per $60-\mathrm{mm}$ culture dish, based on preliminary measurements to determine the optimal cell concentration for each irradiation dose. The culture dishes were kept in a light-protected humidified chamber to avoid activation of 5-ALA-induced PpIX. Cells in the 5-ALA group were incubated in complete medium containing $1 \mathrm{mM}$ 5-ALA for $4 \mathrm{~h}$ and then washed with PBS. After the medium was replaced with $3 \mathrm{ml}$ of fresh culture medium, cells were irradiated by a $\gamma$-irradiator (Gammacell 40 Extractor; Nordion International, Inc., Kanata, Ontario, Canada) at a dose-rate of $0.8 \mathrm{~Gy} / \mathrm{min}$. During ionizing irradiation, culture dishes were kept in a dark container at room temperature. Control cells were treated with the same procedure as those in the 5-ALA group but without exposure to 5-ALA. The response of the cells to ionizing irradiation was evaluated by standard colonyforming assay. After 12 days of irradiation treatment, cells were fixed and stained using a Diff Quick Staining kit (Sysmex Co., Kobe, Japan). Three or four culture dishes were prepared for each dose point and the experiment was performed three times, independently. Only colonies containing $\geq 50$ cells were scored. Plating efficiency was determined for unirradiated controls treated in the same way and maintained under the same conditions. The surviving fraction was then calculated. Radiation survival curves were plotted as logarithms of the surviving fraction against radiation dose and analyzed using the single-hit multi-target model (16). The $37 \%$ survival dose $\left(\mathrm{D}_{0}\right.$ value) was calculated from the slope of the exponential cell-killing line.

Evaluation of the response of cells to multi-dose ionizing irradiation. The 9L gliosarcoma and C6 glioma cells were seeded at a density of 100 cells ( 0 Gy) and 400 cells (total 8 Gy), respectively, per 60-mm culture dish. Cells in the 5-ALA group were incubated with complete medium containing $1 \mathrm{mM}$ 5-ALA for $4 \mathrm{~h}$ and then washed with PBS. After the medium was replaced with $3 \mathrm{ml}$ of fresh culture medium, cells were exposed to ionizing irradiation ( $2 \mathrm{~Gy} / \mathrm{day}, 0.8 \mathrm{~Gy} / \mathrm{min}$ ), and incubated in the dark. The next day, cells were treated with 5-ALA and exposed to ionizing irradiation again. This procedure was performed for 4 consecutive days. Thus, cells were exposed to ionizing irradiation for a total of $8 \mathrm{~Gy}(2 \mathrm{~Gy} / \mathrm{day}$, 4 days). Cells in the control group were treated with the same procedure as those in the 5-ALA group but without exposure to 5-ALA. After 8 days of irradiation, cells were fixed and stained. The surviving fraction was then calculated as previously described.

Subcellular localization of 5-ALA-induced PpIX in glioma cells in vitro. Cells were seeded into $35-\mathrm{mm}$ glass-bottom dishes (Asahi Techno Glass Co., Tokyo, Japan) in fresh medium containing $1 \mathrm{mM}$ 5-ALA and incubated in the dark at $37^{\circ} \mathrm{C}$ for $4 \mathrm{~h}$. Cells were then washed with PBS and examined by confocal laser scanning microscopy (LMS5 Pascal; Zeiss)
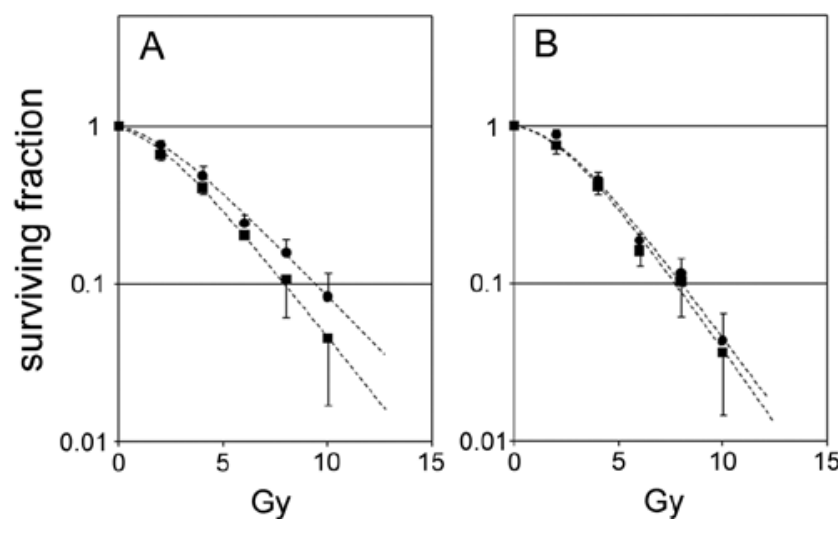

Figure 1. Radiation dose-response curves of (A) 9L gliosarcoma and (B) C6 glioma cells. The symbols and $\bullet$ represent cells pre-treated with $1 \mathrm{mM}$ of 5-ALA for $4 \mathrm{~h}$ before ionizing irradiation and cells pre-treated without 5-ALA, respectively. Results represent the means of 3 independent assays, each performed in 3 or 4 culture dishes at each dose point. Error bars indicate standard deviation (SD).

with excitation/emission wavelength set at 488/560 nm with a long-pass filter, respectively.

Detection of intracellular reactive oxygen species (ROS) in glioma cells in vitro. Intracellular ROS production was detected using the oxidant-sensitive fluorescent probe DCFD with a confocal laser scanning microscope $(17,18)$. Cells were seeded into 35-mm glass-bottomed dishes in fresh medium containing $1 \mathrm{mM} 5$-ALA and incubated in the dark at $37^{\circ} \mathrm{C}$ for $4 \mathrm{~h}$. After the incubation, cells were washed with PBS twice, incubated with $10 \mu \mathrm{M}$ DCFD for $10 \mathrm{~min}$, washed with PBS and exposed to $10 \mathrm{~Gy}$ of ionizing irradiation. DCF fluorescence (excitation, $488 \mathrm{~nm}$; emission, 505- to 530-nm band-pass filter) and 5-ALA-induced PpIX (excitation, $488 \mathrm{~nm}$; emission, 560-nm long-pass filter) was imaged on a confocal laser scanning microscope (LMS5 Pascal, Zeiss). All procedures were performed in the dark.

Statistical analyses. The data are presented as mean \pm SD and were analyzed with Fisher's protected least significant difference. Significance was defined as $\mathrm{P} \leq 0.05$.

\section{Results}

5-ALA-induced PpIX-mediated response of cells to singledose ionizing irradiation. Fig. 1 shows the enhanced effect of 5-ALA-induced PpIX on the radiosensitivity of exponentially growing 9L and C6 cells to single-dose ionizing irradiation. The error bars on the survival curves indicate standard deviation calculated from 3 independent experiments. Based on irradiation dose-response curves corresponding to the multitarget single-hit model, 9L cells treated with 5-ALA before single-dose ionizing irradiation showed higher radiosensitivity $\left(\mathrm{D}_{0}\right.$ value, $\left.4.35 \pm 0.20 \mathrm{~Gy}\right)$ than control cells $\left(\mathrm{D}_{0}\right.$ value, $4.84 \pm 0.23 \mathrm{~Gy})(\mathrm{P} \leq 0.05)$ (Figs. $1 \mathrm{~A}$ and 2$)$. However, there were no significant differences in the response of C6 cells to single-dose ionizing irradiation between the 5-ALA treated group and the control $\left(\mathrm{D}_{0}\right.$ value, $4.26 \pm 0.10$ and $4.51 \pm 0.17 \mathrm{~Gy}$, respectively) $(\mathrm{P}=0.096)$ (Fig. $1 \mathrm{~B})$. 


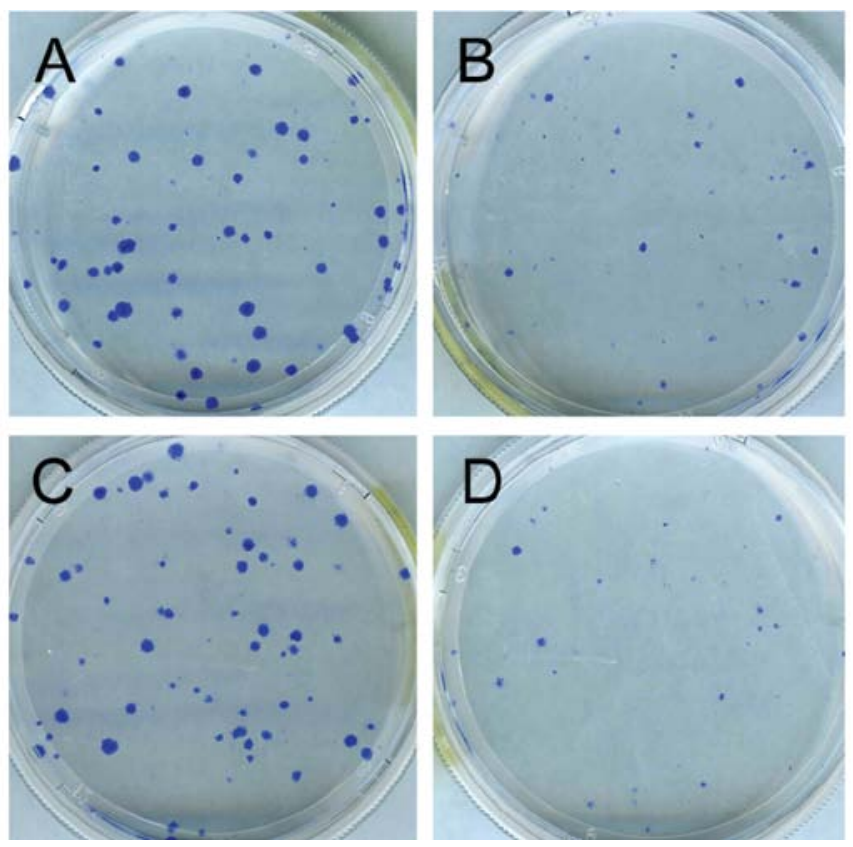

Figure 2. Representative culture plate of standard colony forming assay in 9L gliosarcoma cells. (A, B) Cells without 5-ALA before ionizing irradiation; (C, D) cells pre-treated with $1 \mathrm{mM}$ 5-ALA before ionizing irradiation. $(\mathrm{A}, \mathrm{C})$ and $(\mathrm{B}, \mathrm{D})$ represent control $(0 \mathrm{~Gy})$ and ionizing irradiation $(8 \mathrm{~Gy})$, respectively. Colonies were fixed and stained using Diff Quick Staining kit.

Cumulative radiosensitizing effect of 5-ALA to multi-dose ionizing irradiation. To evaluate the cumulative radiosensitizing effect of 5-ALA, glioma cells were exposed to multi-dose ionizing irradiation and the number of colonies were counted. Cells treated with 5-ALA in each irradiation revealed higher radiosensitivity in both 9L and C6 cells, compared to control $(\mathrm{P} \leq 0.05)$ (Fig. 3).

Subcellular localization of 5-ALA-induced PPIX in glioma cells. Fig. 4 shows that 5-ALA-induced PpIX was located mainly in the cytoplasm in both 9L and C6 cells. However, the fluorescence intensity of PpIX in 9L cells was markedly higher than that of C6 cells (Fig. 4).

ROS production in glioma cells after ionizing irradiation. Intracellular ROS generated by ionizing radiation in $9 \mathrm{~L}$ cells were visualized by DCF fluorescence using the oxidantsensitive probe DCFD (Fig. 5). Our imaging condition of confocal laser scanning microscopy, as previously described, did not detect an interaction between 5-ALA-induced PpIX and DCF fluorescence (Fig. 5B and E). DCF fluorescence was concentrated in the cytoplasm of glioma cells after ionizing irradiation. Pre-treatment of cells with 5-ALA before irradiation caused an increase in DCF fluorescence (Fig. 5J). Interestingly, DCF fluorescence generated by ionizing irradiation predominantly coincided with 5-ALA-induced PpIX in the cytoplasm of 9L cells (Fig. 5L). In addition, PpIX fluorescence decreased slightly after ionizing irradiation (Fig. 5I), compared to controls without ionizing irradiation (Fig. 5A). Although C6 cells were subjected to the same imaging methods, DCF fluorescence in these cells was very faint (data not shown).
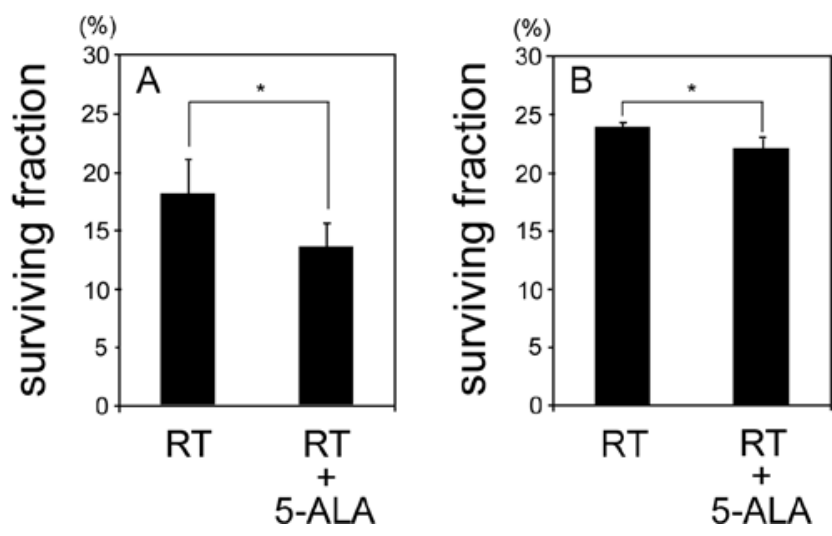

Figure 3. Cumulative radiosensitizing effect of 5-ALA induced by multi-dose ionizing irradiation. (A) 9L gliosarcoma, (B) C6 glioma. Irradiation (RT) + 5-ALA. Cells were pre-treated with 5-ALA and exposed to ionizing irradiation for 4 consecutive days. RT, irradiation only. The total dose of ionizing irradiation was $8 \mathrm{~Gy}$ in each group. Bars, $\mathrm{SD},{ }^{*} \mathrm{P} \leq 0.05$.

\section{Discussion}

5-ALA-induced PpIX as a specific radiosensitizer in glioma cells. Enhancement of tumour responses to ionizing irradiation by 5-ALA-induced PpIX in glioma cells was confirmed using a standard colony-forming assay in vitro. The present data demonstrate that 5-ALA-induced PpIX affects the radiosensitivity of glioma cells with variation according to the cell line irradiated. Porphyrin compounds, in particular HpD and Photofrin, have been found to act as efficient radiosensitizing agents for tumours in vitro and in vivo $(10,11,13,14)$. Recent studies reported the efficacy of gadolinium texaphyrin (Gd-Tex), which is a pentadentate aromatic metalloporphyrin, identified as a tumour-selective sensitizer for ionizing irradiation in clinical studies $(19,20)$. Previous reports showed low intracellular concentrations and a weak radiosensitising effect of 5-ALA-induced PpIX in response to single-dose ionizing irradiation compared to HpD and Photofrin $(12,14,15)$. However, the present results suggest that multi-dose ionizing irradiation enhances the radiosensitizing effect of 5-ALA-induced PpIX, and thus inhibits cell proliferation in glioma cells in vitro (Fig. 3). These data indicate that 5-ALA-induced PpIX has a radiosensitizing effect in gliomas and is appropriate for multidose ionizing irradiation.

Potential mechanism of the radiosensitizing effect of 5-ALAinduced PpIX. The mechanism underlying the radiosensitizing effect of 5-ALA-induced PpIX has not been elucidated to date. In the present study, differences in the fluorescence intensity of intracellular 5-ALA-induced PpIX were observed between 9L and C6 cells (Fig. 4). In 9L cells, high intracellular PpIX concentrations correlated with enhanced inhibition of cell proliferation by ionizing irradiation compared to C6 cells. These results suggest that the radiosensitizing effect depends on the intracellular concentration of P PIX, which concurs with other reports (14). We confirmed the production and localization of intracellular ROS induced by ionizing irradiation in glioma cells pre-treated with 5-ALA using confocal laser scanning microscopy in vitro. Irradiation of cells in combination with 5-ALA treatment resulted in the enhanced production of 

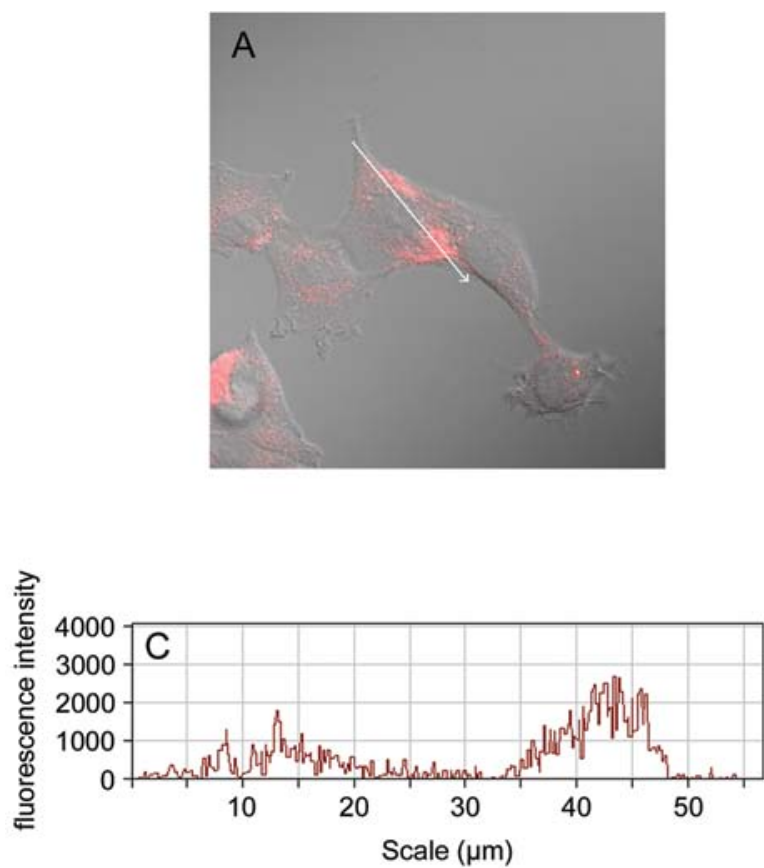
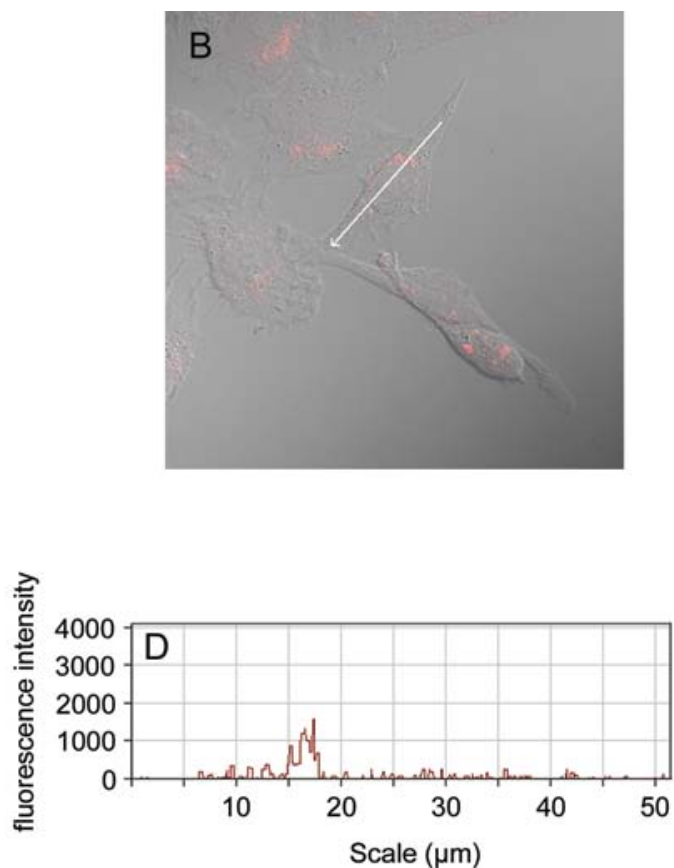

Figure 4. Visualization and quantification of intracellular 5-ALA-induced PpIX in 9L gliosarcoma (A, C) and C6 glioma cells (B, D) in vitro. Micrographs show the fluorescence image of intracellular 5-ALA-induced PpIX merged with the DIC image (A, B). The fluorescence intensity of the micrographs of intracellular 5-ALA-induced PpIX was plotted.

\section{PplX \\ DCFD \\ Merge}
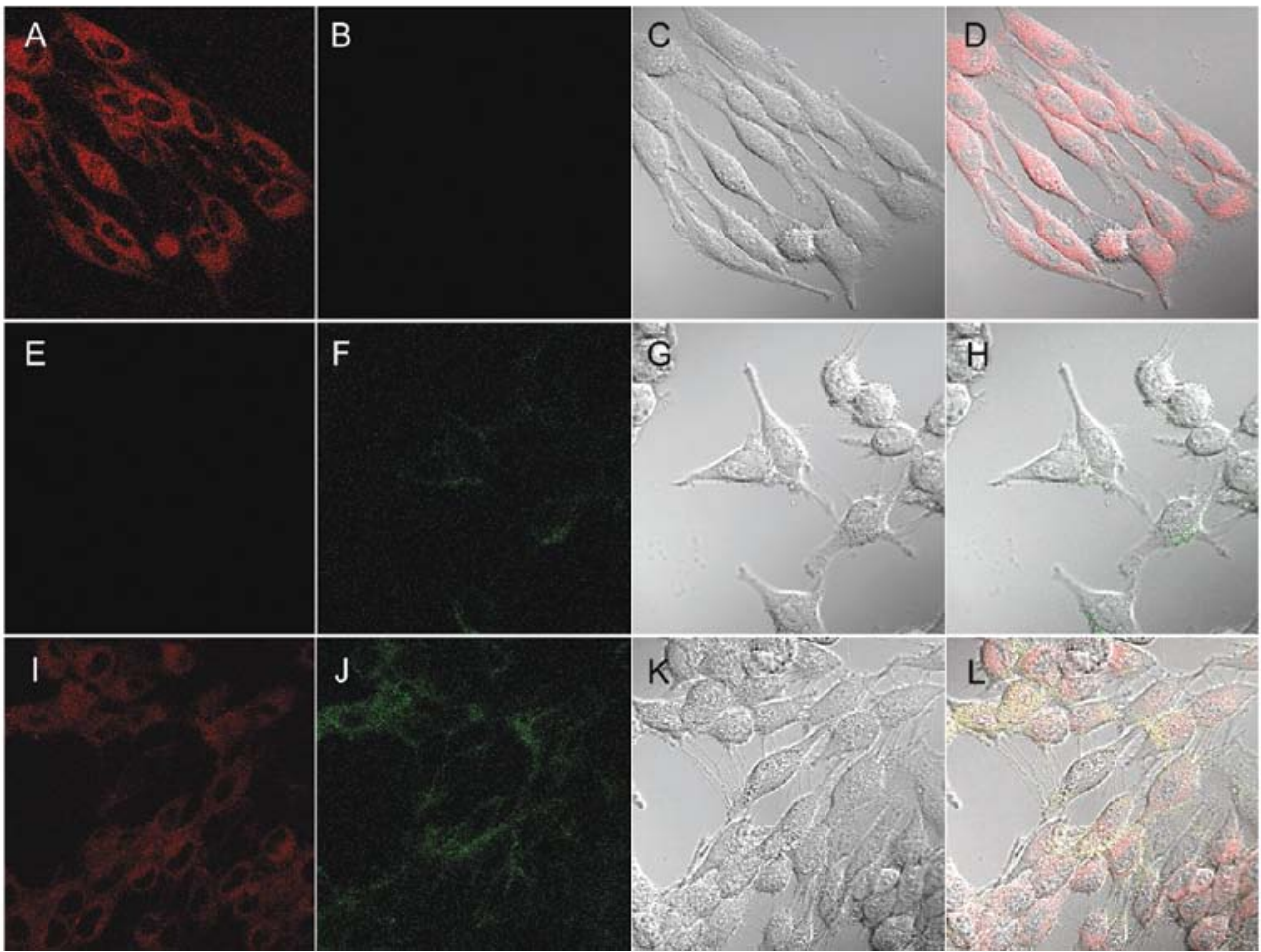

Figure 5. Visualization of intracellular 5-ALA-induced PpIX and ROS induced by ionizing irradiation in 9L gliosarcoma cells in vitro. (A-D) Cells treated with $1 \mathrm{mM} 5$-ALA, no ionizing irradiation. (E-H) Cells pre-treated with $10 \mu \mathrm{M}$ DCFD and then exposed to ionizing irradiation (10 Gy). (I-L) Cells pre-treated with $1 \mathrm{mM}$ 5-ALA and $10 \mu \mathrm{M}$ DCFD and then exposed to ionizing irradiation (10 Gy). (A, E and I) Fluorescence image of intracellular 5-ALA-induced PpIX. (B, F and J) DCF fluorescence image. (C, G and K) DIC image. (D, H and L) Merged image corresponding to the fluorescence of intracellular 5-ALA-induced PpIX, DCF fluorescence and the DIC image. The intracellular localization of 5-ALA-induced PpIX and ROS was analyzed using confocal laser scanning microscopy (fluorescence and DIC images). DCF fluorescence was mainly accumulated in the cytoplasm of glioma cells after ionizing irradiation (F, J). Note that DCF fluorescence increased when cells were treated with 5-ALA and then irradiated (J). Interestingly, DCF fluorescence was predominantly co-localized with 5-ALA-induced PpIX (L) and the fluorescence of intracellular PpIX was reduced after ionizing irradiation (I), compared to control (A). 
intracellular ROS compared to irradiation without 5-ALA in 9L cells. To the best of our knowledge, this is the first report to show the production and intracellular localization of ROS in response to ionizing irradiation concurrent with 5-ALA treatment. In general, the damaging effect of ionizing radiation on living cells is mainly attributed to the effects of ROS, including superoxide, hydroxyl radical and hydrogen peroxide, generated by the decomposition of water. Secondary radicals formed by the interaction of hydroxyl radicals with organic molecules are thought to play an important role in cellular damage $(21,22)$. ROS can damage critical cellular components such as DNA, proteins and lipids, and eventually lead to physical and chemical damage to tissues (23). Some studies speculate that Photofrin may enhance the radiolytic activity by reacting with cytotoxic, activated molecules such as hydroxyl radicals, which are known to be generated as a result of the primary interaction of ionizing radiation with water (24). In the present study, intracellular ROS produced by ionizing irradiation co-localized with 5-ALA-induced PpIX in 9L cells (Fig. 5L). In addition, ionizing irradiation reduced the fluorescence of intracellular PpIX (Fig. 5A and B). There are two possible explanations for this phenomenon: i) intracellular PpIX may produce ROS in response to ionizing irradiation, which would affect its own formation or ii) ROS produced by intracellular PpIX and ionizing irradiation may damage the cell membrane and induce leakage of intracellular PpIX to the extracellular space. Taken together, the present results suggest that intracellular 5-ALA-induced PpIX plays an important role in the production of ROS and the radiosensitizing effect under ionizing irradiation conditions.

We evaluated the radiosensitizing effect of 5-ALA-induced PpIX in different glioma cell lines in vitro. The damaging effect of ionizing irradiation depends on the amount of intracellular ROS produced by intracellular 5-ALA-induced PpIX in glioma cells. Although the radiosensitizing effect of 5-ALA-induced PpIX is weak, multi-dose ionizing irradiation enhances the radiosensitizing effect of 5-ALA-induced PpIX in glioma cells.

\section{Acknowledgements}

This study was supported by a Japanese Ministry of Education, Culture, Sports, Science and Technology Grant-in-Aid for Young Scientists (no. 20791024).

\section{References}

1. Shapiro WR, Green SB, Burger PC, et al: Randomized trial of three chemotherapy regimens and two radiotherapy regimens and two radiotherapy regimens in postoperative treatment of malignant glioma. Brain Tumor Cooperative Group Trial 8001. J Neurosurg 71: 1-9, 1989.

2. Kornblith PL and Walker M: Chemotherapy for malignant gliomas. J Neurosurg 68: 1-17, 1988.

3. Fritsch C, Goerz G and Ruzicka T: Photodynamic therapy in dermatology. Arch Dermatol 134: 207-214, 1998.
4. Mimura S, Ito Y, Nagayo T, et al: Cooperative clinical trial of photodynamic therapy with photofrin II and excimer dye laser for early gastric cancer. Lasers Surg Med 19: 168-172, 1996.

5. Mlkvy P, Messmann H, Pauer M, et al: Distribution and photodynamic effects of meso-tetrahydroxyphenylchlorin (mTHPC) in the pancreas and adjacent tissues in the Syrian golden hamster. Br J Cancer 73: 1473-1479, 1996.

6. Yamamoto J, Hirano T, Li S, et al: Selective accumulation and strong photodynamic effects of a new photosensitizer, ATX-S10. $\mathrm{Na}$ (II), in experimental malignant glioma. Int J Oncol 27: 1207-1213, 2005.

7. Stummer W, Pichlmeier U, Meinel T, Wiestler OD, Zanella F and Reulen HJ: Fluorescence-guided surgery with 5-aminolevulinic acid for resection of malignant glioma: a randomised controlled multicentre phase III trial. Lancet Oncol 7: 392-401, 2006.

8. Muller PJ and Wilson BC: Photodynamic therapy for malignant newly diagnosed supratentorial gliomas. J Clin Laser Med Surg 14: 263-270, 1996.

9. Yamamoto J, Yamamoto S, Hirano T, et al: Monitoring of singlet oxygen is useful for predicting the photodynamic effects in the treatment for experimental glioma. Clin Cancer Res 12: 7132-7139, 2006

10. Kostron H, Swartz MR, Miller DC and Martuza RL: The interaction of hematoporphyrin derivative, light, and ionizing radiation in a rat glioma model. Cancer 57: 964-970, 1986.

11. Kulka U, Schaffer M, Siefert A, et al: Photofrin as a radiosensitizer in an in vitro cell survival assay. Biochem Biophys Res Commun 311: 98-103, 2003.

12. Schaffer M, Ertl-Wagner B, Schaffer PM, et al: Feasibility of photofrin II as a radiosensitizing agent in solid tumors - preliminary results. Onkologie 29: 514-519, 2006.

13. Berg K, Luksiene Z, Moan J and Ma L: Combined treatment of ionizing radiation and photosensitization by 5 -aminolevulinic acid-induced protoporphyrin IX. Radiat Res 142: 340-346, 1995.

14. Luksiene Z, Juzenas $P$ and Moan J: Radiosensitization of tumours by porphyrins. Cancer Lett 235: 40-47, 2006.

15. Schaffer M, Schaffer PM, Jori G, et al: Radiation therapy combined with photofrin or 5-ALA: effect on Lewis sarcoma tumor lines implanted in mice. Preliminary results. Tumori 88: 407-410, 2002.

16. Elkind MM and Sutton H: Radiation response of mammalian cells grown in culture. 1. Repair of X-ray damage in surviving Chinese hamster cells. Radiat Res 13: 556-593, 1960.

17. Akhlynina TV, Jans DA, Rosenkranz AA, et al: Nuclear targeting of chlorin e6 enhances its photosensitizing activity. J Biol Chem 272: 20328-20331, 1997.

18. Ogura S, Yazaki K, Yamaguchi K, Kamachi T and Okura I: Localization of poly-L-lysine-photosensitizer conjugate in nucleus. J Control Release 103: 1-6, 2005.

19. Viala J, Vanel D, Meingan P, Lartigau E, Carde P and Renschler M: Phases IB and II multidose trial of gadolinium texaphyrin, a radiation sensitizer detectable at MR imaging: preliminary results in brain metastases. Radiology 212: 755-759, 1999.

20. Rosenthal DI, Nurenberg P, Becerra CR, et al: A phase I singledose trial of gadolinium texaphyrin (Gd-Tex), a tumor selective radiation sensitizer detectable by magnetic resonance imaging. Clin Cancer Res 5: 739-745, 1999.

21. Hall EJ, Worgul BV, Smilenov L, Elliston CD and Brenner DJ: The relative biological effectiveness of densely ionizing heavy-ion radiation for inducing ocular cataracts in wild-type versus mice heterozygous for the ATM gene. Radiat Environ Biophys 45: 99-104, 2006.

22. Ewing D and Jones SR: Superoxide removal and radiation protection in bacteria. Arch Biochem Biophys 254: 53-62, 1987.

23. Cerutti PA: Prooxidant states and tumor promotion. Science 227: 375-381, 1985.

24. Schaffer M, Schaffer PM, Corti L, et al: Photofrin as a specific radiosensitizing agent for tumors: studies in comparison to other porphyrins, in an experimental in vivo model. J Photochem Photobiol B 66: 157-164, 2002. 